\title{
Accessi vascolari per emodialisisi I perché di alcune scelte
}

\author{
Francesco Quarello
}

\author{
Divisione di Nefrologia e Dialisi, Ospedale San Giovanni Bosco, Torino
}

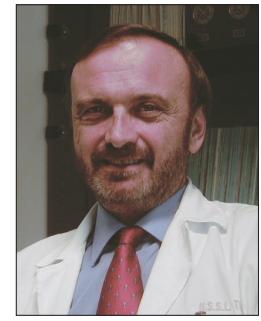

I

problema dell'accesso vascolare riporta alla memoria, in ogni cultore della dialisi, eventi drammatici, che hanno contrassegnato in positivo o in negativo la sua esperienza personale. Ma, al di là di singoli episodi più o meno eclatanti, l'allestimento di un accesso vascolare è una componente routinaria della pratica quotidiana di un Centro dialisi. Anche i pazienti in dialisi peritoneale non sono esenti da questa necessità perché, pur con una depurazione effettuata dal peritoneo, la ruota di scorta di una fistola arterovenosa funzionante è ritenuta in molti Centri un requisito indispensabile.

\section{Perché il nefrologo?}

La consapevolezza della centralità dell'accesso vascolare nell'armamentario terapeutico del paziente uremico ha spinto molti nefrologi, in particolare quelli italiani, a rendersi indipendenti dagli erogatori "ufficiali" delle procedure invasive, i chirurghi vascolari, gli anestesisti, i radiologi interventisti, per garantire un intervento immediato in ogni evenienza, dalla disostruzione di una fistola arterovenosa o di una protesi, al posizionamento di un catetere venoso centrale (CVC).

È difficile sostenere che questa sia la miglior strada percorribile e, soprattutto, che sia valida per tutti i Centri dialisi, anche quelli con un numero limitato di pazienti, ma è fuori di dubbio che la dimestichezza con una procedura aumenta sensibilmente l'attenzione nel prevenirne e risolverne le complicanze. In ogni caso, prescindendo dall'obbligo di acquisirne le specifiche competenze, è opportuno che la cultura degli accessi vascolari si diffonda tra tutti i componenti dell'équipe nefrologica e infermieristica. Il neonato Gruppo di Studio degli Accessi vascolari della SIN ha accolto la sfida con entusiasmo, consapevole della necessità di offrire un approccio uniforme a questa tematica e ha istituito una serie di corsi sugli accessi vascolari capillarmente distribuiti nelle varie Regioni italiane. Il passaggio da una conoscenza di base dei vari tipi di accesso vascolare e delle rispettive complicanze a una padronanza delle tecniche più sofisticate e complesse richiede però un periodo di apprendistato più o meno lungo presso centri chirurgici o nefrologici di riferimento.

La crescente precarietà delle condizioni cliniche dei pazienti incidenti e il prolungarsi della permanenza in dialisi di quelli prevalenti ha reso molto più complesso il quadro dei possibili interventi e ha richiesto un ricorso sempre maggiore, nei casi più complicati, al chirurgo vascolare e al radiologo interventista, che sono in grado di applicare procedure più congrue e invasive, con l'ausilio di tecnologie sofisticate e complesse.

Anche nel nostro Centro, tradizionalmente autonomo nella gestione degli accessi vascolari, da alcuni anni è stata avviata una proficua collaborazione con i chirurghi vascolari, che eseguono alcuni degli interventi più complessi, dalla chiusura di aneurismi di grandi dimensioni al confezionamento di loop protesici sui vasi femorali.

\section{Perché un catetere venoso centrale?}

Paradossalmente, pur disponendo dell'appoggio dei chirurghi vascolari per 
i casi più difficili, negli ultimi anni abbiamo assistito nel nostro Centro al progressivo aumento dei cateteri venosi centrali nei pazienti in dialisi continuativa, tanto che l'attuale prevalenza di CVC nei cronici è di circa il $20 \%$. È una soluzione di comodo o una vera necessità? Ufficialmente, le indicazioni al posizionamento di un catetere venoso centrale restano limitate ai casi che giungono tardivamente alla dialisi o sono reduci dal fallimento di un accesso precedente. In presenza di un albero venoso ancora idoneo al confezionamento di una fistola arterovenosa, il CVC dovrebbe costituire un provvedimento temporaneo ma, in alcuni casi, l'atteggiamento del paziente, soprattutto se anziano e poco propenso ad accettare un nuovo intervento chirurgico, e un'aspettativa di vita limitata, trasformano in pratica la scelta in una soluzione definitiva.

Se il confezionamento di un accesso con vasi nativi o protesici appare impraticabile, per esaurimento dei vasi venosi superficiali o profondi, il catetere venoso centrale costituisce un comodo rifugio, che evita al paziente lo stress di interventi chirurgici ripetuti. In effetti, la protesi in PTFE è gravata da frequenti episodi di stenosi e trombosi. Per il salvataggio di protesi malfunzionanti sono richiesti frequenti interventi di trombectomia e revisione chirurgica, trombolisi meccanica o farmacologica, senza che nessuno di questi provvedimenti si sia dimostrato più efficace dell'altro.

Tuttavia, anche il cateterismo venoso è gravato da un elevato tasso di insuccessi, dovuti soprattutto a infezioni e trombosi, ed è di solito utilizzato come risorsa di ultimo impiego. Il largo ricorso ai cateteri, nonostante l'elevato tasso di fallimenti, riflette il disagio che ancora esiste con tutti i tipi di accessi per emodialisi.

Le linee guida DOQI suggeriscono un limite all'impiego dei CVC del 10\%, sulla base dell'elevata incidenza di infezioni riportata in letteratura, soprattutto in alcuni Centri americani, dove si raggiungono addirittura picchi di 10-15 episodi di infezione per 1000 giorni-paziente. La causa di risultati così negativi risiede nella mancanza di rigorose procedure di asepsi nella fase di posizionamento e in quella di manutenzione dei cateteri. Che adeguate norme di prevenzione possano consentire risultati migliori è dimostrato dalla favorevole esperienza maturata nel nostro Centro su oltre 350 cateteri venosi centrali in vena giugulare nel paziente cronico. Da un'indagine retrospettiva su 37 cateteri di Tesio consecutivamente posizionati a partire dal gennaio 2000 abbiamo registrato 8 episodi di batteriemia, corrispondenti a un'incidenza di 0.5 / 1000 giorni-catetere. Nettamente più frequenti sono le infezioni dell'ostio cutaneo e del tunnel (3.8 episodi/1000 giorni-catetere), che si confermano un problema aperto, meritevole di maggiore attenzione verso nuove soluzioni.

\section{Perché un port sottocutaneo?}

Il port sottocutaneo totalmente impiantabile è un accesso vascolare per emodialisi di recente acquisizione, che si pone in alternativa ai cateteri venosi tunnellizzati. Ai vantaggi di tipo estetico dei port si associa una teorica maggior protezione nei confronti delle infezioni, come confermato dalla nostra esperienza e da quella di altri autori (1-5).

Dal momento della loro introduzione in Italia nel gennaio 2000 abbiamo posizionato nell'Ospedale San Giovanni Bosco 37 port sottocutanei Dialock $^{\circledR}$ (Medival, Padova), alcuni dei quali in pazienti inviati da altri Centri. Data la peculiarità e la "novità" del sistema penso sia utile riferire $i$ dati più salienti della nostra esperienza.

Il dispositivo è stato impiantato dal nefrologo nella sala chirurgica divisionale in anestesia locale, con controllo radiologico del posizionamento della punta del catetere in atrio destro durante l'intervento, senza che siano state segnalate complicanze chirurgiche di rilievo. La durata d'osservazione complessiva è di oltre 500 mesipaziente, con una permanenza media di circa 400 giorni. Il sistema è attualmente utilizzato da 27 pazienti, con una sopravvivenza a un anno del-
1'85\%. In 5 casi il dispositivo ha dovuto essere rimosso, per trombosi della vena centrale ( 2 casi), sepsi ( $1 \mathrm{ca}-$ so), erosione cutanea (1 caso) e leucocitosi persistente in mielodisplasia (1 caso). Tre pazienti sono deceduti per cause non correlate all'uso del Dialock $^{\circledast}$. Nei due anni di osservazione sono stati evidenziati 12 episodi di batteriemia (0.9/1000 giorni-catetere) in 9 pazienti, con un'incidenza non significativamente diversa da quella osservata su un numero equivalente di pazienti portatori di cateteri Tesio inseriti nello stesso periodo $(0.5 / 1000$ giorni-catetere; $p=0.18$ ). Invece, una differenza statisticamente significativa è emersa dall'esame delle infezioni cutanee, nettamente meno frequenti nel gruppo dei port, dove sono state osservate 2 infezioni della tasca $(0.16 / 1.000$ giorni-catetere) rispetto ai 61 episodi di infezione dell'ostio e del tunnel (3.8/1000 giorni-paziente) nei cateteri tunnellizzati tradizionali, trattati quindi con un'antibiotico-terapia più prolungata (24.6 vs 14.3 giorni; p: 0.006).

Un eventuale abbattimento degli indici di batteriemia deriverà dall'impiego di agenti batteriostatici nel liquido di stazionamento dei cateteri venosi centrali. A seguito dei brillanti risultati ottenuti da Sodemann (6) con l'impiego di torolidina associata a citrato, abbiamo iniziato uno studio collaborativo italiano sull'uso del Neutrolin ${ }^{\circledR}$ (Medival, Padova) nel periodo interdialitico nei CVC (7).

L'incannulamento del Dialock, effettuato nell' $80 \%$ delle sedute dialitiche dal personale infermieristico, è stato descritto come facile nel $97.1 \%$ dei casi, difficile nel $2.9 \%$. Non è stato rinviato o sospeso nessun trattamento a causa di problemi di infissione degli aghi.

Tre pazienti sono stati inseriti nel programma di dialisi lunghe notturne (6 ore e 30 minuti) e hanno giudicato il sistema particolarmente vantaggioso durante il sonno, grazie allo stabile e sicuro ancoraggio degli aghi al torace. Un altro paziente, in trattamento emodialitico autogestito da molti anni, ha potuto riprendere la dialisi domiciliare, nonostante il fallimento di nume- 
rosi accessi vascolari sia nativi sia protesici, dopo un breve periodo di addestramento della partner.

\section{Che fare in futuro?}

La nostra esperienza con i cateteri venosi centrali si è dimostrata sostanzialmente soddisfacente e ha contribuito a risolvere situazioni che potevano diventare drammatiche per il paziente. Si tratta ovviamente di dispositivi a rischio, per la possibile insorgenza di trombosi e stenosi delle vene centrali, addirittura mortali se non tempestivamente diagnosticate. Peraltro, a fronte di due casi che hanno avuto esito sfavorevole, negli ultimi 10 anni ne sono stati risolti positivamente quattro dopo immediata rimozione del catetere e avvio della terapia anticoagulante.

In futuro continueremo a utilizzare $i$ vasi nativi tutte le volte che sarà possibile. Se la situazione imporrà il ricorso a tecniche più invasive, sarà data la precedenza a sistemi e procedure che favoriranno la migliore qualità di vita per il paziente, riducendo al minimo le complicanze.

fquarello@pobox.com

\section{BIBLIOGRAFIA}

1. Levin NW, Yang PM, Hatch DA, et al. Initial results of a new access for hemodialysis. Technical Note. Kidney Int 1998; 54: 173945 .

2. Canaud B, My H, Morena M, et al. Dialock ${ }^{\circledR}$ : a new vascular access device for extracorporeal renal replacement therapy. Preliminary clinical results. Nephrol Dial Transplant 1999; 14: 692-8.

3. Canaud B, Levin N, Ing T, My H, Dubrow AJ, Polaschegg HD, Prosl FR. Dialock ${ }^{\circledR}$ : Pilot Trial of a new vascular port access device for hemodialysis. Semin Dial 1999; (12) 5: 382-8.

4. Quarello F, Forneris G, Formica M, et al. Dialock Subcutaneous port for hemodialysis: a twelvemonth experience in a single centre: The Journal of Vascular Access 2001; 2: 73-9.

5. Quarello F, Forneris G, Pozzato M. Prosthetic devices for vascular access. In: Ronco C, La Greca G (eds): First International Course on Hemodialysis Technology. Contrib Nephrol. Basel, Karger, 2002, vol 137 , pp 1-8.

6. Sodemann K, Polaschegg HD, Feldmer B. Two Years' Experience with Dialock and CLS ${ }^{\mathrm{TM}}$ (ANew Antimicrobial Lock Solution). Blood Purif 2001; 19: 251-4.

7. Quarello F, Forneris G. Prevention of hemodialysis catheter related bloodstream infection using an antimicrobial lock. Blood Purif 2002; 20: 87-92. 